\begin{tabular}{|c|c|}
\hline \multirow{3}{*}{ 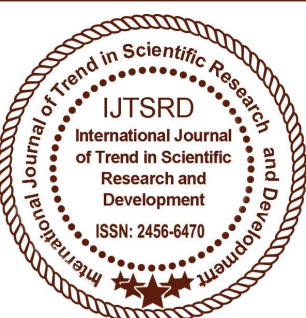 } & $\begin{array}{l}\text { International Journal of Trend in Scientific } \\
\text { Research and Development (IJTSRD) }\end{array}$ \\
\hline & Access Journal \\
\hline & ISSN No: 2456 - 6470 | www.ijtsrd.com | Volume - 2 | Issue -3 \\
\hline
\end{tabular}

\title{
Recognition of Indian Sign Language using SVM classifier
}

\author{
Sudhir S. Kanade \\ Professor \& Head, Electronics \& Telecommunication \\ Engineering Department, TPCT's College Of \\ Engineering, Osmanabad, Maharashtra. India.
}

\author{
Padmanabh D. Deshpande \\ Electronics \& Telecommunication Engineering \\ Department, TPCT's College Of Engineering, \\ Osmanabad, Maharashtra, India
}

\begin{abstract}
Sign language is the medium of communication for the hearing impaired people. It uses gestures instead of sound to convey meaning. It combines handshapes, orientation and movement of the hands, arms or body, facial expressions and lip-patterns for conveying messages.
\end{abstract}

Different types of project are done against deaf, mute, hard hearing people. A system with computer human interface is proposed for sign language recognition. But there is country wide variation available in that project. The main idea of this project is design a system which is useful for communication of that people with outside world in any public places, so that no need to interpreter in public places. In that project we need the isolated images in the form of database with Indian sign language of numeric sign. A regular camera is useful for acquiring this numeric sign. Principal Component Analysis (PCA) is used for preprocessing, in which the removal of redundant and unwanted data is done.

Keywords: PCA , SVM classifier

\section{INTRODUCTION}

Sign language is a language for the people who are challenging in hearing and speech. Despite common misconceptions, sign languages are complete natural languages, with their own syntax and grammar. Signs are made of units referred to as cheremes, which are any of a small set of elements, analogous to the phoneme in speech, proposed as the basic structural units by which the signs of a sign language are represented, and including the hand shapes, hand movements, and locations of the hands in relation to the body employed in a particular sign language. A sign is made of cheremes and any two signs can be differentiated using at least one chereme. There are mainly two different approaches in sign language recognition - Glove based approach and vision based approach. Sign language is helpful with communication between deaf, mute, hard hearing people and normal people. In normally, sign language is understandable for the signer and the person who know the sign language but it is so much difficult for who does not know the sign language or meaning of any gesture. In M. Geetha and U. C. Manjusha [4], the strategy of the Eigenvectors method consists of extracting the characteristic features.

Where the images of Indian sign language can be used as input and the system will display the English alphabet, which the mute, deaf people want to tell. A regular camera is used for acquiring this sign. For developing this project we need numeric sign database with 26 English alphabets sign \& 9

Numeric sign with proper images. In that project every word or every alphabet is assigned with a particular image. This image is in the form of .jpg so that we can easily use the database. These images are in static or dynamic form. Static gestures dynamic form. Static gestures have fixed position of hand whereas dynamic gestures have movement of hands and body parts.

The proposed scheme uses PCA for feature extraction. 
Principal Component Analysis (PCA) (Rafael and Woods, 2002) is a well-known and one of the most successful techniques used in image recognition and compression for extracting feature and representing data. It is technique widely used in the area of pattern recognition, computer vision and signal processing. Principal component analysis (PCA), also known as Karhunen-Loeve expansion, is a classical feature extraction and data representation technique widely used in the areas of pattern recognition and computer vision such as face recognition. The purpose of PCA is to reduce the large dimensionality of the data space (observed variables) to the smaller intrinsic dimensionality of feature space (independent variables), which are needed to describe the data economically. This is the case when there is a strong correlation between observed variables. By discarding minor components, the PCA effectively reduces the number of features and displays the data set in a low dimensional subspace. In this study the feature extraction algorithm based on PCA is chosen. The coefficients of these methods are used as feature vectors which efficiently represent extracted image.

Cloud computing is an emerging technology in the present scenario which enhances enormous data storage and its processing. Various issues are present in maintaining the cloud environment. Some among them include data privacy and data confidentiality faced by the data owners while storing their private data onto the cloud. The domain of Data mining mainly helps in efficient retrieval of the content stored in the public space using various retrieval methods. Generally, in cloud, the data privacy is provided by outsourcing the files in encrypted form before being uploaded. This creates more demand in server side data utilization i.e., searching on encrypted files from the cloud. As of now the retrieval is made productive through keyword based search. Most recent works focus on the single keyword provided as queries in the search process. In the keyword ranked search, it had been difficult to store when the number of keywords are more. In the work carried out below, an efficient and reliable multi-keyword search method called KGram search is developed. Using K-gram it is possible to retrieve more than one file i.e., related files to the keyword searched, productively relative to misspelled keywords too. In addition the user behavior is too monitored from the start for enhancing keyword search and it also ensures proper confidentiality via access confirmation through secret keys. The system thus tends to provide more chances for tackling data privacy and confidentiality issues. It can also achieve sub linear search time thereby increasing the query processing and accuracy in keyword search.

\section{LITERATURE REVIEW}

There are different theories used for Indian Sign Convention presented by different authors. The surveyed literature on Sign Convention is as follows:

In Tamil sign letter Balakrishnan G. Subha Rajam [1], Proposed a method, which was recognizing a 32 set of combinations \& 10 for each up and down position of fingers. This method is used for the up/down position of fingers which is converted into decimal numbers, this number is recognized in to the Tamil alphabet A set of database in the form of images of sizes $640 \times 480$ pixels are captured. Palm image extraction is used to convert RGB to grayscale images. The experiment result is $96.87 \%$.

For static and dynamic alphabet sign Rekha J., Bhattacharya and S. Majumder [2], proposed a system this system was used 23 static ISL alphabet signs from 40 different signers are collected as training samples and 22 videos are used as testing samples. The images are extracted by the method of Principle Curvature Based Region Detector.

Multi class non-linear KNN are used as sign classifiers. The experiment result for static $94.4 \%$ and for dynamics it was $86.4 \%$. In Indonesia northern Bali jungle village of "Bengkala" for generations, where a high percentage of residents are Deaf. Bengkala has a higher than normal deaf-since-birth population for over seven generations. Today, 42 of Bengkala's almost-3,000 villagers have been deaf since birth.

By comparison, about two or three births per 1,000 in the United States produce a deaf or hard of hearing child. The high percentage of deafness is caused by a geographically centric recessive gene, called DFNB3, which has been present in the village for over seven generations. Rather than ostracizing deaf residents, villagers in Bengkala have adapted to a deaf lifestyle. Throughout the village, people speak with their hands. Kata kolok, known as "the talk of the deaf," is a unique, rural sign language, independent of international or Indonesian sign language. 


\section{METHODOLOGY}

The proposed system is shown in the figure 1. If no standard data set is available to experiment on automatic recognition of ISL gestures then Two data sets of ISL character signs are created. First set contains gestures belongs to single handed Indian Sign Language characters and the second set contains double handed gestures of Indian Sign Language. The details of acquiring of data set are given in data set creation section.

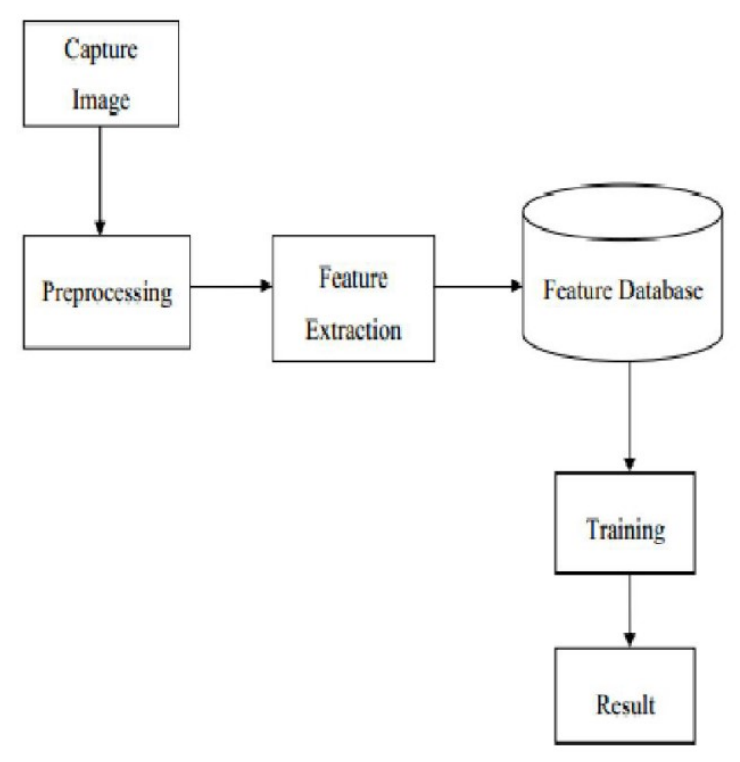

Fig.1 Overview of proposed system

\section{1] Input Sign language Image -}

Two types of dataset are creating for this experiment. In this dataset 26 images of single \& double handed images are captured using digital camera. These images are in the form of 200X300 RGB pixel size. These images are collecting from single mute male person for each character.

We use this image in the JPG format because it is very easy to extract the image in different hardware and software environment. The second dataset created for this experiment are images for each number. The memory required for this dataset is nearly $40 \mathrm{~kb}$. This image is flat rectangular shape in structure.

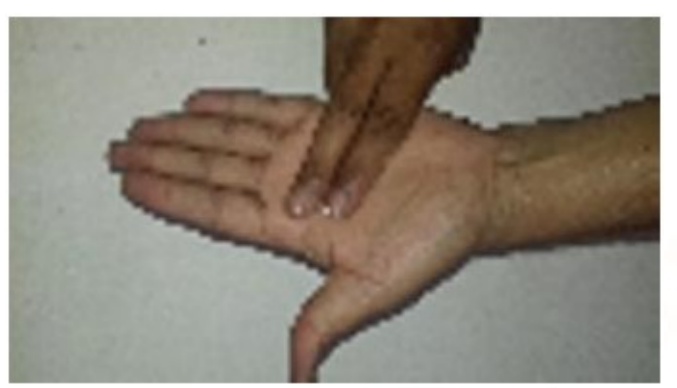

Fig.2 Input image

\section{2] Preprocessing}

After collecting the database from user we need to preprocess those images. Preprocessing images commonly used for removing low-frequency background noise, normalizing the intensity of the individual particles images, Firstly we convert RGB images into grey scale images by using MATLAB (rgb to gray converter). This will convert RGB images to high intensity Grey scale images. In this step we can perform segmentation and noise removal operation.

The main aim of pre-processing is an improvement in input data (sign language images) that data suppresses unwanted distortions. Image preprocessing technique uses the considerable redundancy in images. Neighboring pixel corresponding to one object in real image have adjusted some or similar brightness value. Preprocessing consists of thresholding, erosion and dilation of collected data images.

\section{i. $\quad$ Skin Thresholding :}

Skin detection is used to search for the human hands and discard other skin colored objects for every frame captured from a webcam shown in fig 3. Image thresholding is used for extracting the significant or desired part of an image and removing the unwanted part or noise. The point operator of major interest is called thresholding which selects pixels that have a particular value, or that are within a specified range. With thresholding, the image can be segmented based on the color. This holds true under the assumption that a reasonable threshold value is chosen. A reasonable threshold value is the taken from the histogram of the original image. The point operator helps us to find objects in a picture if the brightness level or range is known. Hence the object's brightness must be known. After detecting skin area for every frame captured, we used contours comparison of that area with the loaded hand postures contours to get rid of other skin like objects exist in the image. If the contours comparison of skin detected area complies with any one of the stored hand gesture contours, a small image will 
enclose the hand gesture area only and that small image will be used for extracting the PCA features.

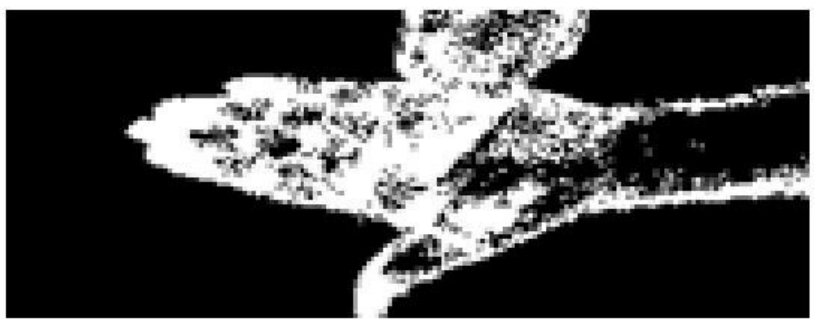

Fig.3 Threshold image

\section{ii. Dilation and erosion :}

Dilation and erosion are basic morphological operations. They are defined in terms of more elementary set operations, but are employed as the basic elements of many algorithms. Dilation is used to increase the object size where as erosion is used to diminish the size of the object. Both dilation and erosion are produced by the moving the mask around the image. The mask which is also called as structuring element or sub image or kernel (Lim, 1990) and (Soille, 2004) and it has both a shape and an origin. The following Eq. 1 and 2 are generally used for dilation and erosion:

Dilation $=\mathrm{X} \perp \mathrm{S}(1)$

Erosion $=X \bar{S}(2)$

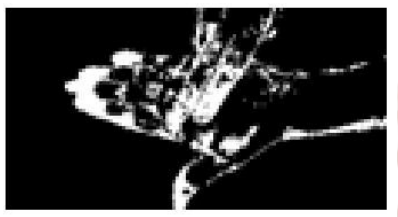

Fig.4 Eroded image

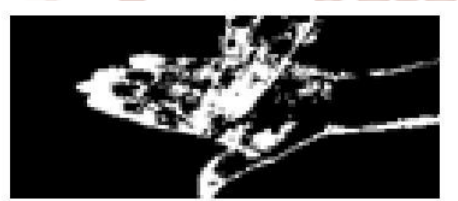

Fig.5 dilated image
Where, $\mathrm{X}$ is an original image and $\mathrm{S}$ is a structuring element.

Based on the image which is to be dilated or eroded the size of the structuring element is choose it is a odd square matrix which contains binary elements that is 0 's and 1's and also the dimension should not exceed the size of the image which is to be processed. The structuring element is a set of coordinates. The origin of the structuring element is the centre element for most cases but it may not be centre point for less cases. The structuring element (Lim, 1990; Soille, 2004) is used to remove the eyelashes and eyelids whose dimension based on region of interest. Following are the examples of structuring elements. In our work we choose the $3 \times 3$ structuring element having its all element as 1 :

\section{$111 \quad 0100110$

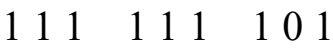

\section{$111 \quad 010 \quad 101$}

The origin of the structuring element identifies the pixel of interest in the input image and all the pixels in its neighborhood are assigned to the corresponding pixel in the output image. The result of Erosion is subtracted from the result of dilation will provide the edge. The following equations are used for dilation and erosion respectively. The result of erosion and dilation is given in Fig. 4 and 5:

\section{3] Feature Extraction -}

The input data which is to be processed is transformed into a reduced representation set of features.

This is referred as feature extraction. Every image consists of large amount of data. This information can be automatically extracted from the images is called as feature extraction.

Here we are using Principal component analysis method for extracting the features.

\section{PCA:}

Linear Discriminant Analysis (LDA), Independent

Component Analysis and PCA are some of the techniques used for feature extraction, among them PCA is powerful method in image formation, Data patterns, similarities and differences between them are identified efficiently.

The other main advantage of PCA is dimension will be reduced by avoiding redundant information, (Daugman,1993) without much loss. Better understanding of principal component analysis is through statistics and some of the mathematical techniques which are Eigen values, Eigen vectors. PCA is a useful statistical and common technique that has found application in fields such as image recognition and compression. Principal Component Analysis (PCA) is a mathematical procedure that uses linear transformations to map data from high dimensional space to low dimensional space. The low dimensional space can be determined by Eigen vectors of the covariance matrix.

\section{PCA Algorithm}

Following are steps involve;

Step 1: Column or row vector of size N2 represents the set of $\mathrm{M}$ images (B1, B2, B3...BM) with size $\mathrm{N}^{*} \mathrm{~N}$ 
Step 2: The training set image average $(\mu)$ is described as

$$
\mu=\frac{1}{m} \sum_{n=1}^{M} B n
$$

Step 3: the average image by vector (W) is different for each trainee image

$$
\mathrm{Wi}=\mathrm{Bi}-\mu
$$

Step 4: Total Scatter Matrix or Covariance Matrix is Calculated from Ö as shown below:

$$
\mathrm{C}=\sum_{\mathrm{n}=1}^{\mathrm{M}} \mathrm{wnwnt}=\mathrm{AAT},
$$

Where $A=[\mathrm{W} 1 \mathrm{~W} 2 \mathrm{~W} 3 \ldots \mathrm{Wn}]$

Step 5: Measure the eigenvectors UL and Eigen values ëL of the covariance matrix $\mathrm{C}$.

Step 6: For image classification, this feature space can be utilized. Measure the vectors of weights

Whereby,

$$
\Omega \mathrm{T}=\left[\mathrm{w} 1, \mathrm{w} 2, \ldots, \mathrm{wM}^{\prime}\right] \text {, }
$$

$$
\mathrm{Hk}=\mathrm{UkT}(\mathrm{B}-\mu), \mathrm{k}=1,2, \ldots, \mathrm{M}^{\prime}(5)
$$

\section{4] Feature Vector -}

In machine learning and pattern recognition feature vector is a n-dimensional vector consist of numerical vector which represent some object. Many algorithm requires feature numerical representation of object. When representing the image feature value shows the pixel of image or whole object in images. Feature vector are equivalent to vector of variable used in linear process. It is just a vector consisting of multiple element or feature. Examples of features are color component length, area, circularity, grey level intensity, magnitude, direction it's depend on which feature is useful for application.

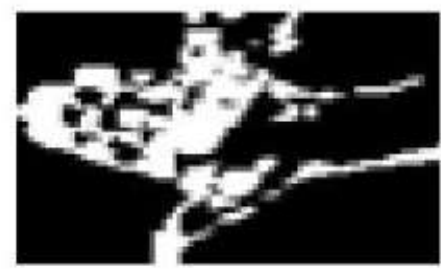

Fig.6 Extracted image

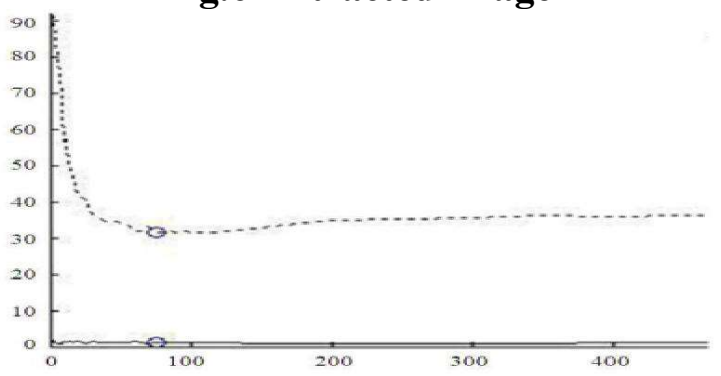

Fig. 7: PCA Performance Evaluation

\section{5] SVM Classifier -}

There are many types of classifier Technique available we use SVM classifier to identify the image. SVM is a support vector machine used for supervised learning model with associated algorithm that analyzed data used for classification and analysis by using Extracted features. SVM classifier is the method of performing the classification task. The support vector machine searches for the closest path which we call "support Vector". Once it found the closest point then the SVM draw a line for connecting them. The support vector machine then declares best separating line which bisect and perpendicular to connecting line. We perform classification by finding the hyper plane for differentiate between two classes. Support vector machine are simply the coordinate of individual observation.

\section{RESULTS AND DISCUSSIONS}

The data set divided into two groups, one used for training and other for testing. The training set consists of $70 \%$ of the aggregate data and remaining $30 \%$ are used as testing means for training we are using images captured from 6 different people and for testing images captured from 2 different people numeric as well as alphabets. We also perform experiments on same $(30 \%$ or $70 \%)$ dataset which is training as well as testing for SVM classifier. The overall accuracy of the proposed method as given below Total No. of Patterns - No. of false result Patterns

$$
\begin{aligned}
& \text { Accuracy }=\text { Total No. of Patterns }- \text { No. of false result Patterns } \\
& 100 \% \\
& \text { Total No. of Patterns }
\end{aligned}
$$

From total 67 testing images 64 images are correctly recognized so the accuracy of this proposed method 95.52\% using PCA features and SVM classifier. 
International Journal of Trend in Scientific Research and Development (IJTSRD) ISSN: 2456-6470

\begin{tabular}{|c|c|c|c|}
\hline Image & $\begin{array}{l}\text { Accuracy } \\
\text { in } \%\end{array}$ & Image & $\begin{array}{l}\text { Accuracy in } \\
\%\end{array}$ \\
\hline & $100 \%$ & & $66.67 \%$ \\
\hline & $100 \%$ & & $100 \%$ \\
\hline & $100 \%$ & & $100 \%$ \\
\hline & $66.67 \%$ & & $66.67 \%$ \\
\hline
\end{tabular}

Table1. Accuracy of Sign language V. CONCLUSION

This Recognizing system is capable of Recognizing Alphabetical and numerical sign with high accuracy using SVM (Support Vector Machine) Classifier. The experimental result is shown that the system is used as "working System" in regular ISL Recognition.

\section{FUTURE SCOPE}

The system can be useful for ISL Static alphabetical and numerical sign. The system is not useful for complete system. For complete system we have to include ISL word or sentences in future. Also other feature extraction algorithms like Wavelet transform, Invariant moments, Shape lets descriptors and other existing methods can be included in conducting experiments for improvement in the results. Other classifiers like Principal Component Analysis (PCA) and Linear Discriminant Analysis (LDA) or a combination of these classifiers can be included in conducting experiments to improve the recognition rate.

\section{REFERENCES}

1. Balakrishna G. and P. S. Rajam. 2012. Recognition of Tamil Sign Language Alphabet using image processing to aid Deaf-Dumb People. International Conference on Communication Technology and System Design. 30: 861-868.

2. Rekha J., J. Bhattacharya and S. Majumder. 2011. Shape, texture and local movement hand gesture features for Indian Sign Language recognition. 3rd International Conference on Trends in Information Sciences and Computing (TISC). : 30-35

3. Divya Deora and Nikesh Bajaj, "Indian sign language recognition", 1st International Conference on Emerging Technology Trends in
Electronics Communication and Networking (ET2ECN), 2012, pp.1-5.

4. M. Geetha and U. C. Manjusha, "A Vision Based Recognition of Indian Sign Language Alphabets and Numerals Using B-Spline Approximation”, International Journal on Computer Science and Engineering (IJCSE), vol. 4, no. 3, 2012, pp. 406415

5. Rohit Sharma, Yash Nemani, Sumit Kumar, Lalit Kane, Pritee Khanna, Member, IAENG WCE 2013, July 3 - 5, 2013, London, U.K. "Recognition of Single Handed Sign Language Gestures using Contour Tracing Descriptor".

6. "Study Of Vision Based Hand Gesture Recognition Using Indian Sign Language" Archana S. Ghotkar and Dr. Gajanan K. Kharate Pune Institute of Computer Technology, Department of Computer Engineering, University of Pune, Pune, India. Feb. 14, 2014 International Journal On Smart Sensing And Intelligent Systems VOL. 7, NO. 1, MARCH 2014

7. Madhuri Sharma, Ranjna Pal and Ashok Kumar Sahoo, ARPN Journal of Engineering VOL. 9, NO. 8, AUGUST 2014 "Indian Sign Language Recognition Using Neural Networks And Knn Classifiers"

8. Ashok K Sahoo, Gouri Sankar Mishra and Kiran Kumar Ravulakollu, "Sign Language Recognition: State of the Art", ARPN Journal of Engineering and Applied Sciences, vol. 9, no. 2, 2014, pp. 116134.

9. Ashok Kumar Sahoo, Kiran Kumar Ravulakollu Department of Computer Science and Engineering, Sharda University, Greater Noida, India $30^{\text {th }}$ September 2014. Vol. 67 No.3 JATIT \& LLS on "Vision Based Indian Sign Language Character Recognition"

10. Zeshan U., M. M. Vasishta and M. Sethna. 2005.Implementation of Indian Sign Language in Educational Settings. Asia Pacific Disability Rehabiliation Journal. (1): 16-40.

11. Sakshi Goyal, Ishita Sharma and Shanu Sharma, "Sign Language Recognition System For Deaf And Dumb People",International Journal of Engineering,vol.2, no. 4, 2013,pp. 382-387.

12. Sahoo, A. K., G. S. Mishra and K. K. Ravulakollu. 2014. Sign Language Recognition: State Of The Art. ARPN Journal of Engineering and Applied Sciences.9(2) : 116-134 